\title{
A Comparative Study of Efficacy of Povidone Iodine Versus Super Oxidized Solution in Lower Limb Ulcers
}

\author{
Nyna Sindhu Akula ${ }^{1}$, Navaneeth Krishna ${ }^{2}$, Sreedhar Sathu ${ }^{3}$ \\ ${ }^{1}$ Department of General Surgery, AIIMS, BIBInagar, Yadadri Bhuvanagiri, Telangana, India, \\ 2. Department of General Surgery, Siddhartha Medical College, Vijayawada, Andhra Pradesh, India. \\ ${ }^{3}$ Department of Orthopaedics, Siddhartha Medical College, Vijayawada, Andhra Pradesh, India.
}

\section{ABSTRACT}

\section{BACKGROUND}

Wounds and their management are important in the practice of surgery. Super oxidised solution is a recent concept in wound management. It is an aqueous solution which is electrochemically processed which is non-toxic, non-irrigating and is having a neutral pH. Povidone iodine is the most common topical wound care product used in surgical practice. Both are affordable solutions for the patients. There are very few studies comparing the efficacy. In this study, we wanted to compare the efficacy of super oxidised solution and povidone iodine in the management of lower limb ulcers.

\section{METHODS}

A prospective study was conducted on 100 patients who were randomized into two groups. Group A was treated with super oxidized solution and Group B were treated with povidone iodine. Assessments of wounds were done on various days $(1,3,5,7$, $9,12,15,18,21)$. Various outcomes of data were analysed using statistical analysis.

\section{RESULTS}

The average percentage reduction in wound size on day 21 was $47 \%$ in Group A compared to $24 \%$ in Group B. Early appearance of granulation tissue, disinfection, decrease in wound size, and less duration of hospital stay was achieved in Group A.

\section{CONCLUSIONS}

The results were more favourable towards super oxidised solution than povidone iodine. In this comparative study, super oxidized solution had faster efficacy and wound healing in patients than povidone iodine.
Corresponding Author:

Dr. Akula Nyna Sindhu,

Scientist Colony, Habsiguda,

Hyherabad - 500044, India.

E-mail: nynasindhukula@gmail.com

DOI: $10.14260 /$ jemds/2021/654

How to Cite This Article:

Akula NS, Krishna N, Sathu S. A comparative study of efficacy of povidone iodine versus super oxidized solution in lower limb ulcers. J Evolution Med Dent Sci 2021;10(37):3220-3224, $10.14260 / \mathrm{jemds} / 2021 / 654$ DOI:

Submission 16-06-2021,

Peer Review 09-08-2021,

Acceptance 16-08-2021,

Published 13-09-2021.

Copyright (C) 2021 Nyna Sindhu Akula et al. This is an open access article distributed under Creative Commons Attribution License [Attribution 4.0 International (CC BY 4.0)]

\section{KEY WORDS}

Super Oxidized Solution, Ulcer, Povidone Iodine. 


\section{BACKGROUND}

Wounds and their management are most commonly encountered in the practice of surgery. The prevalence of leg ulceration is approx. $1-2 \%$. Their origin is multifactorial. ${ }^{1}$ Thus, a proper diagnosis of their aetiologies like arterial, venous, and neuropathic ulcers is essential to salvage the limb. Ulcers may appear similar, but on closer inspection, they are completely different entities. Not only the lower limb ulcers are of different aetiologies but also have specific anatomic locations that themselves give rise to specific problems in their management. An ulcer is a disruption or break in the continuity of the covering epithelium - skin or mucous membrane, which may be due to the molecular death of the surface epithelium or its traumatic removal. Ulcer can be classified clinically as a spreading ulcer, healing ulcer and a callus ulcer. The management of ulcer includes the investigations to know the aetiology of ulcer and treatment. A thorough workup, including vascular, metabolic, and physical aspects, is essential.

The principles of ulcer management are: To determine the aetiology, precise assessment of the ulcer, identifying and correction of the associated comorbidities, debridement of ulcer, avoiding adherent dressings, lifestyle modification and appropriate pharmacotherapy.

Newer interventions in wound healing are MicroRNA and wound healing, integrins, stem cells, negative pressure. miRNAs are small non-coding RNA molecules that play a pivot role in wound healing. Understanding the dysregulated miRNAs in chronic wounds will therefore enable the development of newer treatments. Chronic non-healing ulcers will have changes in the cell receptors called integrins. Integrins are bidirectional signalling receptors for extracellular matrix that regulates keratinocyte mediated changes to the wound microenvironment. The inhibition of integrin receptors will provide better healing of chronic wounds. The stem cell therapy to promote the regeneration of skin is utilized by the regenerative medicine to aid in better wound healing. Vacuum-assisted wound closure: - It is a therapeutic technique done using a suction dressing to remove excess exudation and promote healing in acute and chronic wounds.

According to World Health Organization (WHO), for every half a minute, a lower limb is amputated due to diabetes in which the woeful thing is that approximately $50 \%$ of all these amputations could have been prevented with basic medical care and awareness. ${ }^{2}$ Also, with the advent of newer diagnostic techniques and surgical techniques, there is resumption of interest in lower limb ulcer management. Newer wound dressings are occlusive dressing, non-occlusive dressing, absorptive dressing, negative suction wound therapy, and skin substitutes.

An ideal wound care product apart from controlling the infection should not affect the normal wound healing process, thus protecting the normal host tissues. Povidone iodine is the most common topical wound care product used in surgical practice and has been effectively used over the years in the treatment of lower limb ulcers and other wounds. ${ }^{3,4}$ Super oxidised solution is a newer concept in wound management. 5,6 Both are affordable solutions for the patients of this study. Leg ulcers are reported to have a virtual impact on every aspect of daily life: pain is present, sleep is impaired, mobility and work capacity is restricted to a certain amount, and personal finances are affected. Chronic leg ulcers are usually associated with significant morbidity, high cost of healthcare, loss of productivity and reduced quality of life. ${ }^{7}$ As wounds are great burdens on the healthcare system, hence the need for this study.

\section{Objectives}

This study was undertaken to compare the super oxidised solution and povidone iodine in the management of lower limb ulcers to observe regarding wound disinfection, decrease in wound size, appearance of granulation tissue and duration of hospital stay.

\section{METHODS}

This was a prospective, randomized, non-blinded, singlecentred comparative study. Patients with lower limb ulcers admitted at Siddhartha Medical College / Govt. General Hospital in General Surgery Department were screened and selected for this study. The study was conducted over a period of 2 years from November 2018 to November 2020. The ethical committee of institute has approved the study and a detailed informed consent was taken from the patient.

\section{Inclusion Criteria}

Age: > 18 years, Traumatic ulcers, Diabetic ulcers, Acute ulcers, Patients willing to give consent.

\section{Exclusion Criteria}

Age: $<18$ years, Ischaemic ulcers, Venous ulcers, Malignant ulcers, Decubitus ulcers, Trophic ulcers, Osteomyelitis of bone, Chronic renal failure, Patients with autoimmune disorders, Patients on long term use of cytotoxic drugs / corticosteroids, Immunosuppression, Cardiac risk patients, Patients not willing to give consent.

\section{Randomization}

A prospective purposive sampling was done with estimated sample size for the study being 100 patients. The patients who fulfilled the eligibility criteria were randomized using a minimization procedure. This randomization was done using a software program called minim, developed by Stephan Evans. Subjects were allocated into two groups.

Variables taken into consideration in randomization were

1. Age with two categories $(<50 />50$ years $)$ with a score, weighted 5.

2. Diabetes mellitus with two categories (present/absent), with a score, weighted 10 .

3. Sex with two categories (male/female), with a score, weighted 2 .

4. Wound size with two categories $(<15 \mathrm{cms} />15 \mathrm{cms})$, with a score, weighted 5 and

5. Grading of ulcer (Grade 1 to 4) with score, weighted 5.

After receiving the screening checklist, the random allocation to two groups was done using simulation 
programme. Group A - Received dressing with topical super oxidized solution. Group B - Received dressing with povidone iodine.

\section{Patient Assessment and Data Collection}

Included patients were subjected to-

1. Detailed clinical history

2. General physical examination and local ulcer examination.

3. Investigations

4. Routine blood investigations - Complete blood picture, renal function tests, liver function tests, blood sugars.

5. Radiological X - ray wherever indicated.

6. Culture sensitivity (C / S) of a swab from an ulcer on Day 1 in all patients, and followed by culture sensitivity on variable days.

7. Venous and arterial doppler scan of the involved limb.

8. Informed written consent

9. Assessments of wounds were done on various days $(1,3$, $5,7,9,12,15,18,21$ )

Various assessment tools were used to compare the wound healing between patients in Group A and Group B based on - Wound size, appearance of granulation tissue, resolution of peri wound erythema and oedema, organisms isolated from culture and sensitivity swabs, procedures done (skin grafting / debridement / fasciotomy / no procedure), Duration of hospitalization. Antibiotics were given to all patients, both intravenous and oral route based on the wound parameters.

\section{Data Analysis}

Data were transcribed onto Excel file, and SPSS (Statistical package for social sciences) software version 25 for windows was used to perform both descriptive and inferential analysis. Frequencies were reported for distribution of categorical variables, and independent sample t-test $\&$ chi-square test was performed for any differences among super oxidised solution (SOS) and povidone iodine (PI) group.

\section{RESULTS}

The mean age in Group A is (56.47 \pm 14.23$)$, and Group B is (53.71 \pm 17.12$)$. (figure 1$)$. In Group $A$, there were 38 males and 12 female patients. 12 patients in Group $A$ and 11 patients in Group B had history of trauma in the form of thorn prick and road traffic accidents. In both groups, the most common position of ulcer is dorsum of the foot, followed by other areas, but some patients had more than one location of the ulcer.

The severity of the diabetic foot ulcers was graded according to the Meggitt - Wagner classification.

Out of 61 diabetic patients,

- 31 patients had grade 1 ulcer - superficial diabetic ulcer.

- 17 patients had grade 2 ulcers with extension involving muscle, tendon, and fascia.

- 13 patients had grade 3 ulcers with abscess underneath it.

In Group A, the average percentage reduction in wound size on Day 21 was $47 \%$ in Group A compared to $24 \%$ in Group B. (table 1)

The mean duration for the day of the appearance of granulation tissue in Group A (fig 5) was (4.84 \pm 1.65 days), whereas the mean duration for the day of the appearance of granulation tissue in Group B (fig 6) was (6.68 \pm 1.98 days). The mean duration for the day of peri wound erythema and oedema resolution was ( $4.72 \pm 1.46$ days) in Group A and (7.5 \pm 1.88 days) in Group B.

The mean duration for the day of wound disinfection (culture negative) was ( $8.56 \pm 2.53$ days) in Group A, whereas the mean duration for the day of wound disinfection (culture negative was ( $12.2 \pm 3.62$ days) in Group B. The mean duration of hospitalization was (12.96 \pm 3.05 days) in Group A, whereas the mean duration of hospitalization was $(15.84 \pm 3.91$ days $)$ in Group B.

The most common organism isolated on culture and sensitivity took on day 1 from all the patients was Staphylococcus aureus, which was present in 27 patients, Pseudomonas in 15 patients, and there is no growth of any organism in 8 patients. Some of the patients had growth of one or more organisms on culture and sensitivity.

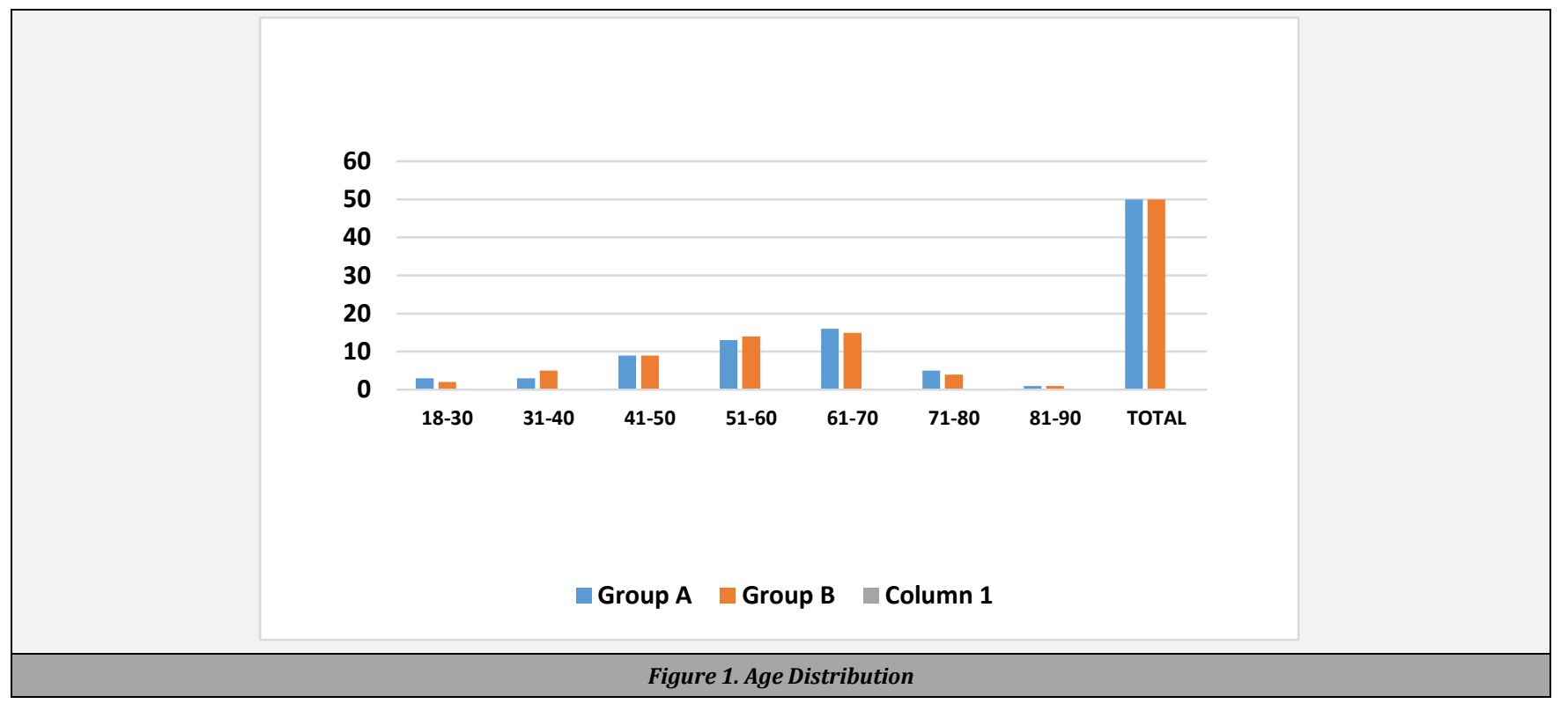




\begin{tabular}{|c|c|c|c|c|c|c|c|}
\hline $\begin{array}{l}\text { Intervention } \\
\text { Group }\end{array}$ & $\begin{array}{l}\text { Wound Size Day } 1 \\
\text { (in cms) }\end{array}$ & $\begin{array}{l}\text { Wound Size Day } \\
9 \text { (in } \mathrm{cms} \text { ) }\end{array}$ & $\begin{array}{l}\text { Wound Size Change } \\
\text { d1 - d9 (in cms) }\end{array}$ & $\begin{array}{l}\text { Wound Size Day } 12 \\
\text { (in } \mathrm{cms} \text { ) }\end{array}$ & $\begin{array}{l}\text { Wound Size Change } \\
\text { d1 - d12 (in cms) }\end{array}$ & $\begin{array}{l}\text { Wound Size Day } \\
21 \text { (in cms) }\end{array}$ & $\begin{array}{c}\text { Wound Size } \\
\text { Change } \mathrm{d} 1-\mathrm{d} 21 \text { (in } \\
\mathrm{cms} \text { ) }\end{array}$ \\
\hline Group B (PI) & 16.724 & & \multirow{3}{*}{0.7} & 8.675 & & 13.442 & 3.793 \\
\hline Mean $\pm S D$ & \pm 5.627 & 14.7 & & \pm 2.055 & $2.625 \pm 1.788$ & \pm 4.372 & \pm 1.584 \\
\hline $\mathrm{N}$ & 50 & 1 & & 4 & & 45 & \\
\hline Group A (SOS) & 15.434 & 5.762 & \multirow{3}{*}{$2.071 \pm 0.268$} & & 4.6 & & \\
\hline Mean \pm SD & \pm 5.93 & \pm 2.991 & & $12.26 \pm 2.497$ & \pm 2.564 & $9.865 \pm 3.703$ & $6.293 \pm 2.359^{* * *}$ \\
\hline $\mathrm{N}$ & 50 & 7 & & 5 & & 38 & \\
\hline \multicolumn{8}{|c|}{ Table 1. Wound Size } \\
\hline
\end{tabular}

Among both the Groups, 58 patients had no surgical intervention (58\%). 16 patients underwent debridement, 7 patients in Group A (SOS), and 9 patients in Group B (PI). 2 patients in group B underwent disarticulation, whereas no patient underwent disarticulation in group A. 7 patients underwent fasciotomy, 4 patients in Group $\mathrm{A}$, and 3 patients in Group B. 17 patients underwent split skin grafting (SSG), 12 patients in Group A, and 5 patients in Group B. In Group A (SOS), 12 patients underwent SSG before 21 days. In Group B (PI), 5 patients underwent SSG before 21 days.

\section{DISCUSSION}

In the current study, the effect of super oxidised solution vs. povidone iodine in the management of lower limb ulcers in each group consisting of 50 patients was studied with standard protocols and methods. This study had revealed good results not only in treating the lower limb ulcers but also in avoiding most of the lower limb amputations. Many variables regarding the wound were compared between the Group A and Group B patients. The patients treated with super oxidised solution (SOS) in Group A showed a rapid decrease in wound size compared to the patients treated with povidone iodine (PI) Group B, comparing the wound sizes from Day 1 to Day 21 . There was a statistically significant reduction in wound size in Group A with an average reduction in size being $47 \%$, whereas the average reduction in wound size was $24 \%$ in Group B patients, which was compared with other studies like Vanita Kapur et al. ${ }^{8}$ P K Pandey et al. ${ }^{9}$ and R Kantly et al. (table 2)

\begin{tabular}{|c|c|c|}
\hline Study & Group A & Group B \\
\hline Vanita Kapur et al. ${ }^{8}$ & $70 \%$ & $50 \%$ \\
\hline PK Pandey et al. ${ }^{9}$ & $71 \%$ & $51 \%$ \\
\hline R Kantly et al. & $58 \%$ & $40 \%$ \\
\hline Present study & $47 \%$ & $24 \%$ \\
\hline
\end{tabular}

Patients treated with super oxidised solution, Group A, had earlier appearance of granulation tissue, earlier resolution of peri wound erythema and oedema and earlier wound disinfection. The results were significant with $P$ value $<0.001$.

The standard of "Twisted Dressing with Super Oxide Solution " was authoritatively begun in the year 2003 when it accomplished a status of "Disinfectant and Antiseptic" in its country Mexico. A few studies have demonstrated the adequacy of the super oxidized preparations and its wide scope of utilizations on few sorts of wounds.

A study of Kapur V, et al. in Amritsar during 2008 to assess the impact and examination of super oxidized arrangement and povidone iodine in various kinds of wounds revealed that super oxidized arrangement was protective and viable in a wide range of wounds. ${ }^{8}$ No systemic and local hypersensitive indications were noted.

A study of Chowdary PS, et al. in Bihar was done to compare super oxidized solution and povidone iodine solution in management of infected diabetic ulcers showed that topical super oxidized solution dressings accelerated the healing process resulting in faster recovery through reduction in ulcer area in patients infected with diabetic ulcers compared to topical povidone iodine dressing. ${ }^{10}$ Tanaka et al. study on antimicrobial property of super oxidized solution stated that it is low cost but affective antimicrobial. ${ }^{11}$ Gutierrez in his study to explore various applications of super oxidised solutions concluded that the moistening effects and minimum toxicity found with the use of this super oxidised solution made it a good choice for wound care management.12,13 G. D. Bakhshi et al. concluded in their study that super oxidised solution is efficacious than povidone iodine in treatment of diabetic ulcers. ${ }^{14}$

The role of topical wound care management in the case of ulcers is crucial. The infection of ulcers can have serious consequences. Presently, infected ulcers are being managed by local dressing with agents like hydrogen peroxide, povidone iodine, EUSOL, local antibiotics, with each having their limitations, and interestingly, none of these dressings is the gold standard in the management of the ulcers. There has always been a search for an ideal antiseptic that is rapidly lethal to all forms of bacteria and their spores, capable of bactericidal property for a prolonged period with no ill effect on host tissues.

Super oxidised solutions have shown to be both safe and efficient as a wound care product that moistens, lubricates, debrides and reduces the microbial load of various types of lesions. ${ }^{15}$ These are electrochemically processed aqueous solutions manufactured from pure solutions rich in reactive oxygen species with neutral $\mathrm{pH}$ and long half-life. It has bactericidal, viricidal, fungicidal and sporicidal properties. ${ }^{16,12}$ Povidone iodine is a stable complex of polyvinyl pyrrolidine and elemental iodine. It kills eukaryotic and prokaryotic cells through iodination of lipids and oxidation of cytoplasmic and membrane compounds. It can stain surrounding structures and has a tendency to kill human cells rarely.

\section{CONCLUSIONS}

In patients treated with super oxidised solution, compared to patients treated with povidone iodine, there was: Enhanced appearance of granulation tissue, enhanced appearance of epithelialization with early resolution of peri wound erythema and oedema. There was also rapid decrease in wound size and early wound disinfection with lesser hospital stay. In diabetic patients, there is increased time taken for the appearance of granulation and epithelialization, and also there is delayed 
resolution of peri wound oedema and erythema, and also the time taken for wound disinfection was delayed.

This study further strengthens the concept of wound healing with this effective mode of treatment, concerning the efficacy of super oxidised solution in different types of wounds, which were previously managed with the conventional method of treatments like povidone iodine. Hence, it is an excellent choice for the management of lower limb ulcers.

Data sharing statement provided by the authors is available with the full text of this article at jemds.com.

Financial or other competing interests: None.

Disclosure forms provided by the authors are available with the full text of this article at jemds.com.

\section{REFERENCES}

[1] Rahman GA, Adigun IA, Fadeyi A. Epidemiology, etiology and treatment of chronic leg ulcer: experience with sixty patients. Annals of African Medicine 2010;9(1):1-4.

[2] Reiber GE, Boyko E, Smith DG. Lower extremity ulcers and amputations in individuals with diabetes. In: Harris MI, Cowie CC, Stern MP, et al. eds. Diabetes in America. $2^{\text {nd }}$ edn. Washington, DC, U.S.: Govt. Printing Office 1995: p. 409-27.

[3] Hilton JR, Williams DT, Beuker B, et al. Wound dressings in diabetic foot disease. Clin Infect Dis 2004;39(Suppl 2):S100-3.

[4] Edmonds M, Foster A. The use of antibiotics in the diabetic foot. Am J Surg 2004;187(5A):25S-8S.

[5] O'Meara SM, Cullum NA, Majid M, et al. Systematic review of antimicrobial agents used for chronic wounds. Br J Surg 2001;88(1):4-21.

[6] Gonzalez-Espinosa D, Perez-Romano L, Guzman-Soriano B, et al. Effects of pHneutral, super - oxidized solution on human dermal fibroblastsinvitro. Int Wound Journal 2007;4(3):241-50.

[7] Gupta SK, Shukla VK. Leg ulcers in the tropics. International Journal of Lower Extremity Wounds 2002;1(1):58-61.

[8] Kapur V, Marwaha AK. Evaluation of effect of superoxidised solution (Oxum) V/S povidine iodine. Indian Journal of Surgery 2011;73(1):48-53.

[9] Pandey PK, Koushariya M, Shukla S, et al. Outcomes of superoxide solution dressings in surgical wounds: a randomized case control trial. Int J Biol Med Res 2011;2(4):965-8.

[10] Chaudhary PS, Kumari S, Singh R. Comparative evaluation of super - oxidized solution and povidone iodine solution in management of infected diabetic ulcers. International Journal of Health and Clinical Research 2020;3(10):13641.

[11] Tanaka H, Hirakata Y, Kaku M, et al. Antimicrobial activity of super oxidized water. J Hosp Infect 1996;34(1):43-9.

[12] Gutiérrez AA. The science behind stable, superoxidized water. Exploring the various applications of superoxidized solutions. Wounds 2006;18(Suppl 1):7-10.

[13] Wolvos TA. Advanced wound care with stable, super oxidized water. A look at how combination therapy canoptimizewound healing. Wounds 2006;18(Suppl 1):11-13.

[14] Bakhshi GD, Joshi G, Bakhshi RG. A comparative study of efficacy of superoxidized solution against povidone iodine in the treatment of diabetic ulcers. International Journal of Science and Research 2020;9(5):746-51.

[15] Abhyankar S, Venkatesh V, Karnad S, et al. Efficacy and safety of oxum in treatment of chronic wounds. Journal of Indian Medical Association 2009;107(12):904-6.

[16] Anand A. Comparative efficacy and tolerability of oxum against povidone iodine topical application in the post caesarean section wound management. Indian Med Gazette 2007: p. 498-505. 